\title{
Treatment of Locally Advanced/Metastatic Colorectal Cancer
}

\author{
Presented by Alan P. Venook, MD, and Christopher G. Willett, MD
}

\begin{abstract}
Few treatment advances have been observed in recent years for the treatment of advanced colorectal cancer (CRC). The goal remains to find approaches beyond FOLFOX and bevacizumab that will prolong remission. Immunotherapy for patients with microsatellite instability-high tumors represents progress, but this is a very small subset and approximately $30 \%$ of patients will not experience response. In locally advanced CRC, good long-term outcomes and manageable toxicity are being achieved with contemporary treatment strategies. Total neoadjuvant therapy, which incorporates induction or consolidation chemotherapy, has improved the treatment of patients with rectal cancer and is now a standard of care, although optimal sequencing is still being debated. Nonoperative management is an emerging option for sphincter preservation, and ongoing studies are evaluating the omission of radiation in select patients.

J Natl Compr Canc Netw 2021;19(5.5):617-621 doi: $10.6004 /$ jnccn.2021.5014
\end{abstract}

"The introduction of FOLFOX and bevacizumab in the early 2000s raised our hopes that progress would be rapid and definitive. Although patients with advanced disease now live longer than in the past-many for $\geq 3$ yearsprogress has largely stalled except for the development of immunotherapies in a small subset of patients with advanced colorectal cancer [CRC]," according to Alan P. Venook, MD, The Madden Family Distinguished Professor of Medical Oncology and Translational Research, and Chief, Gastrointestinal Oncology Clinical Program, University of California San Francisco, and Associate Director, Program Development, UCSF Helen Diller Family Comprehensive Cancer Center, who spoke at the NCCN 2021 Virtual Annual Conference. "For almost 20 years, FOLFOX + bevacizumab has remained the mainstay of treatment. While this may not be optimal first-line treatment for all patients, approximately $75 \%$ to $80 \%$ of patients start with this regimen," he said. "We have made progress-but it's been grudging, and it's important to emphasize that not every patient has benefited from the progress ... . How do we do better?"

One way to make progress in treatment is to identify more biomarkers, such as those targeted for testing by NCCN: RAS mutations, BRAFV600E mutations, HER2 amplification, microsatellite instability (MSI), and rightsidedness of tumors. Unfortunately, there are no biomarkers yet to predict efficacy for "our central drugs," bevacizumab and oxaliplatin, he pointed out. Other ways are to expand the use of immunotherapies and to harness artificial intelligence to better understand the disease.

Turning to the treatment of locally advanced CRC, Christopher G. Willett, MD, Professor, and Chairman, Department of Radiation Oncology, Duke University
Medical Center and Duke Cancer Institute, explained how contemporary and future strategies are aiming to provide excellent long-term outcomes while minimizing toxicity, maximizing patient satisfaction, and, ideally, preserving the anal sphincter. To this end, the standard of care of patients with rectal cancer is now total neoadjuvant therapy (TNT), and nonoperative management (ie, wait and see) is an emerging option and the omission of radiotherapy (RT) is being studied in select patients.

\section{The Good News: Immunotherapy for MSI-High Tumors}

The link between tumors that are MSI-high (MSI-H) or mismatch repair deficient (dMMR) has clearly changed the treatment paradigm for patients in this category. "We hope to find MSI-H status in every patient with advanced CRC, though it's a small minority-only 5\% to 7\%," Dr. Venook said. ${ }^{1}$

Initial studies of the immune checkpoint inhibitors (ICIs) pembrolizumab and nivolumab ( \pm ipilimumab) showed "dramatic" benefit for MSI-H/dMMR tumors., 2 Subsequent studies have validated the clear efficacy of these drugs in MSI-H tumors. Dr. Venook suggested that moving ICIs to the first-line setting, and identifying ways to make them effective in patients with microsatellite stable tumors, could help improve overall survival (OS) in metastatic CRC. There is also an urgent need, he added, to understand why approximately $30 \%$ of patients with MSI-H tumors do not experience response to immunotherapy and to study ways to change their response.

For patients with MSI-H/dMMR tumors, the NCCN Clinical Practice Guidelines in Oncology (NCCN Guidelines) for Colon and Rectal Cancers list pembrolizumab 
or nivolumab \pm ipilimumab as preferred regimens for those able to undergo intensive therapy. Single agents are preferred in patients not suitable for intensive therapy, and combination nivolumab/ipilimumab has a category $2 \mathrm{~B}$ recommendation in these patients.

In the nonrandomized CheckMate 142 study, investigation nivolumab + ipilimumab in the first-line setting, at 29 months follow up for the overall population, median OS was not reached and the 24-month OS rate was $79 \%{ }^{4}$ In the randomized KEYNOTE-177 trial, ${ }^{5}$ median progression-free survival was 16.5 months with pembrolizumab versus 8.2 months with chemotherapy (hazard ratio [HR], $0.60 ; P=.0002$ ). Median duration of response was not reached with pembrolizumab and was 10.6 months with chemotherapy. These recommendations are in the NCCN Guidelines.

\section{Immunotherapy or Chemotherapy First?}

The findings from KEYNOTE-177 raise an important issue, Dr. Venook stated, based on the observation that in the study's first 6 months the chemotherapy arm actually fared better. ${ }^{5}$ This finding was substantiated in a subsequent analysis that showed some patients who started chemotherapy experienced disease progression, crossed over to the pembrolizumab arm, and still experienced worse outcomes than those initially treated with the ICI. ${ }^{6}$

"The survival curve still favors the initial immunotherapy population, with a median OS that has not been reached. In my mind, this argues that most patients with MSI-H CRC should get an ICI first-line," Dr. Venook said. The caveat, he added, is that the optimal sequence is not certain, and chemotherapy can still be a good option for many patients with MSI-H tumors. This was shown in an analysis of the CALGB/SWOG 80405 population, in which bevacizumab conveyed a 30-month median OS in those with MSI-H disease. " "This reminds us that chemotherapy in MSI-H disease may still be appropriate. Even though pembrolizumab is much more active and is favored in terms of quality of life, it's important to consider whether the patient may progress rapidly and not get a chance to move on to chemotherapy."

The recommendation, therefore, is that an ICI is preferred as first-line therapy except for patients with large-volume disease, in whom a 2- to 3-month delay in chemotherapy could be detrimental.

\section{Other Actions}

The NCCN Guidelines now recognize that TAS-102 (eg, trifluridine/tipiracil), an oral nucleoside similar to 5-FU, may be beneficial in chemorefractory metastatic CRC. In a 2020 study of TAS-102 \pm bevacizumab, the combination reduced the risk of disease progression (HR, $0.45 ; P=.0015)$ and death (HR, 0.55; $P=.028$ ) over single-agent TAS-102. ${ }^{8}$ Based on this, TAS-102 ( \pm bevacizumab) has moved up in
NCCN Guidelines as an option for patients previously treated with oxaliplatin and irinotecan, in the first, second, or third line. "I score this as a somewhat promising result," he commented.

\section{Next Steps May Involve Artificial Intelligence}

Dr. Venook said ongoing research is evaluating targeted combinations in BRAF-mutant disease in the first-line setting (ANCHOR trial; ClinicalTrials.gov identifier: NCT03693170) and ICIs + chemotherapy in the adjuvant setting (ATOMIC trial; NCT02912559). Investigators are also searching for biomarkers in MSI-H tumors that might predict response to treatment.

Efforts are also underway to develop and expand the use of "big data" and artificial intelligence. A recently published study used a machine learning approach to validate a 67-gene signature, based on $>220,000$ real-world and clinical trial patients, to accurately predict outcomes from first-line oxaliplatin-based chemotherapy in advanced CRC. ${ }^{9}$ "Although we use FOLFOX routinely, it's worth knowing which patients may not derive benefit, because FOLFOX is associated with neuropathy," Dr. Venook said. Although the data are preliminary and must be validated, if the gene signature is valid, it could identify patients for whom a regimen other than FOLFOX would be better.

Dr. Venook has personally been involved in a machine learning approach to discerning why rightsidedness is associated with worse survival and prognosis, shown in CALGB/SWOG 80405. ${ }^{10}$ Turning to "the artificial intelligence realm," investigators identified potential biomarkers that helped explain the gene signatures of these tumors, and concluded that right-side location of the primary tumor is not a direct driver of OS but more of a surrogate for its molecular features, and these are related. For example, odds ratios for worse survival were 3.5 for the angiogenesis/extracellular matrix (ECM) remodeling gene signature and 7.2 for $B R A F$ mutations; these features were significantly more prevalent in right-sided tumors. Study investigators are now applying the FIRE2 dataset to this algorithm, looking to explain the effect of bevacizumab on right-sided tumors. "All of this is exciting, but only if it pans out. Our goal is to use this kind of mechanism to get beyond FOLFOX/bevacizumab," he said.

\section{TNT Now Standard of Care}

The traditional approach to locally advanced rectal cancer is a "sandwich approach," wherein the patient undergoes long-course chemoradiotherapy (LCCRT) or short-course RT (SCRT) followed by surgery, then recovers to receive adjuvant chemotherapy. Due to some of the challenges with administering adjuvant chemotherapy in the postoperative setting, there has been a great deal of interest in and investigation of TNT, Dr. Willett said. SCRT is given as 25 Gy in 5 fractions without concurrent 
chemotherapy, with surgery performed within days of completion or 4 to 8 weeks later. LCCRT typically includes 50.4 Gy given over 5.5 weeks with concurrent chemotherapy, with surgery performed about 6 weeks later.

TNT optimizes the delivery of trimodality therapy by incorporating chemotherapy before or after radiationbased approaches. The two approaches are (1) chemotherapy first, followed by LCCRT or SCRT and then surgery, or (2) LCCRT or SCRT first, followed by chemotherapy, then surgery. The relative merit of these approaches is an active area of investigation.

The rationale for using TNT is to improve compliance, offer better tolerability, enhance downstaging of the primary tumor, facilitate organ preservation (through nonoperative management), provide earlier treatment of micrometastatic disease, and decrease the interval from ileostomy to reversal. Potential drawbacks include delays in surgical resection (possibly potentiating local progression), and the development of toxicity that may impact the possibility of definitive resection or lead to worsening performance status and nutritional status.

The phase II TIMING trial studied patients with stage II/III rectal cancer within $12 \mathrm{~cm}$ of the anal verge. ${ }^{11}$ It evaluated 4 approaches, all beginning with continuousinfusion 5-FU + RT, followed by a 4- to 6-week rest; 3 arms then received modified FOLFOX6 for 2, 4, or 6 cycles. Patients underwent total mesorectal excision (TME). Adjuvant FOLFOX was recommended postsurgery to complete a total of 8 cycles. The complete pathologic response rate was highest for the arm receiving 6 cycles of modified FOLFOX6 (38\%) and dropped to $18 \%$ for those receiving zero cycles of this regimen $(P=.004)$.
The zero-cycle group also had significantly worse disease-free survival (DFS) than patients receiving modified FOLFOX6, but OS was not significantly different, nor were grade $\geq 3$ surgical complications.

Dr. Willett described recent clinical trials that support TNT as a standard of care (Table 1). ${ }^{12-15}$ In these studies, TNT led to significantly higher rates of pathologic complete response, 3-year DFS rates, and 3-year TME-free survival, as well as lower 3-year disease-related treatment failure rates, thus meeting the various primary endpoints of these studies. With no loss of efficacy in DFS or OS, TNT is also better tolerated and associated with improved compliance. It may be especially optimal for patients with highrisk features.

Upcoming trials should resolve questions regarding sequencing of chemotherapy and CRT, and the relative merit of TNT over the sandwich approach, Dr. Willett said. He noted that the RAPIDO study demonstrated improved outcomes for patients undergoing TNT and surgery compared with adjuvant chemotherapy after CRT and surgery. ${ }^{14}$

\section{How NCCN Guidelines Incorporate TNT}

The NCCN Guidelines for Rectal Cancer therefore actively incorporate the TNT strategy. This is recommended for T3 disease with any nodal status and clear circumferential margins, and T1-2,N1-2 disease. For T3 disease of any nodal status and clear circumferential margins, TNT strategies can initiate chemotherapy or CRT first, then the alternate treatment. The same is true for more advanced disease, such as T4 disease, locally unresectable disease, or lesions approaching the circumferential

\section{Table 1. Recent Total Neoadjuvant Therapy Trials}

\begin{tabular}{|c|c|c|c|c|}
\hline Study & Patients, $\mathrm{n}$ & Eligibility & Trial Design & Primary Endpoint \\
\hline CAO/ARO/AIO- $12^{12}$ & 311 & $\begin{array}{l}\text { cT3 }<6 \mathrm{~cm} \text { from AV } \\
\text { cT3 ( }>5 \mathrm{~mm} \mathrm{ES}): 6-12 \mathrm{~cm} \mathrm{AV} \\
\text { cT4 } \\
\text { All } \mathrm{N}+\end{array}$ & $\begin{array}{l}\text { FOLFOX (3 cy) } \rightarrow \text { CRT } \rightarrow \text { TME } \\
\text { vs } \\
\text { CRT } \rightarrow \text { FOLFOX ( } 3 \text { cy }) \rightarrow \text { TME }\end{array}$ & $\begin{array}{l}\text { pCR: } \\
25 \% \\
17 \%\end{array}$ \\
\hline PRODIGE $23^{13}$ & 461 & $\begin{array}{l}\text { cT3N0 } \leq 10 \mathrm{~cm} \text { from AV } \\
\text { cT1-T3N }+ \\
\text { Circumferential margins }>2 \mathrm{~mm}\end{array}$ & $\begin{array}{l}\mathrm{mFOLFIRINOX} \rightarrow \mathrm{CRT} \rightarrow \mathrm{TME} \rightarrow \mathrm{ChT} \\
\text { vs } \\
\mathrm{CRT} \rightarrow \mathrm{TME} \rightarrow \mathrm{ChT}\end{array}$ & $\begin{array}{l}\text { 3-year DFS: } \\
75.7 \% \\
68.5 \%\end{array}$ \\
\hline RAPIDO $^{14}$ & 912 & $\begin{array}{l}\text { cT4 a/b } \\
\text { EVI } \\
\text { N2 } \\
\text { Mesorectal fascia + } \\
\text { enlarged lateral LN }\end{array}$ & $\begin{array}{l}\mathrm{SCRT} \rightarrow \text { CAPOX/FOLFOX }(18 w k) \rightarrow \text { TME } \\
\text { vs } \\
\text { CRT } \rightarrow \text { TME } \rightarrow \text { CAPOX/FOLFOX } 24(w k)\end{array}$ & $\begin{array}{l}\text { 3-year dis-rel tx failure: } \\
23.7 \% \\
30.4 \%\end{array}$ \\
\hline OPRA $^{15}$ & 306 & $\begin{array}{l}\text { Distal lesions (APR or ColoA) } \\
\text { CS II or III }\end{array}$ & $\begin{array}{l}\text { CRT } \rightarrow \text { ChT } \rightarrow \text { Restaging (CR) } \\
\text { vs } \\
\text { ChT } \rightarrow \text { CRT } \rightarrow \text { Restaging (CR) } \\
(\mathrm{ChT:} 4 \text { mo mFOLFOX6 or CAPOX) }\end{array}$ & $\begin{array}{l}\text { 3-year TME-free: } \\
59 \% \\
43 \%\end{array}$ \\
\hline
\end{tabular}

Abbreviations: APR, abdominoperineal resection; AV, anal verge; CAPOX, capecitabine/oxaliplatin; ChT, chemotherapy; ColoA, coloanal anastomosis; CR, complete response; CRT, chemoradiotherapy; CS, clinical stage; cy, cycles; DFS, disease-free survival; dis-rel tx, disease-related treatment; ES, extramural tumor spread; EVI, extramural vascular invasion; FOLFOX, fluorouracil/leucovorin/oxaliplatin; LN, lymph node; mFOLFIRINOX, modified oxaliplatin/irinotecan/leucovorin/ fluorouracil; mFOLFOX, modified FOLFOX; pCR, pathologic complete response; SCRT, short-course radiotherapy; TME, total mesorectal excision. 
Table 2. Nonoperative Management

\begin{tabular}{|lccccc|}
\hline Study & Patients, $n$ & cCR & Follow-Up & Local RFS & DFS \\
\hline Habr-Gama et al ${ }^{16}$ (Brazil) & $90 / 183$ & $49 \%$ & 60 mo & $69 \%$ & $68 \%$ \\
\hline Martens et al ${ }^{17}$ (Netherlands) & $61 / 100$ & $61 \%$ & $41.1 \mathrm{mo}$ & $84.6 \%$ & $80.6 \%$ \\
\hline Appelt et al ${ }^{18}$ (Danish) & $40 / 55$ & $73 \%$ & 23.9 mo & $84.5 \%$ & - \\
\hline
\end{tabular}

Abbreviations: cCR, complete clinical response; DFS, disease-free survival; RFS, recurrence-free survival.

resection margin. Starting with CRT improves pathologic complete response (and thereby downstaging) versus starting with chemotherapy, so it makes sense to start with CRT in patients with more advanced disease where margin is an issue.

\section{Watch and Wait Nonoperative Management}

The nonoperative approach termed "watch and wait" involves treating patients with LCCRT, then evaluating disease response. Pivotal early studies showed local recurrence-free survival rates of $69 \%,{ }^{16} 85 \%,{ }^{17}$ and $85 \%,{ }^{18}$ and DFS rates of $68 \%{ }^{16}$ to $81 \%$ (ClinicalTrials.gov identifier: NCT00952926) (Table 2).

New data are currently being reported from the retrospective International Watch \& Wait Database (IWWD) of 793 patients who achieved complete clinical responses after CRT and were managed by observation (ie, watch and wait). ${ }^{19}$ Overall, the 5-year local regrowth-free survival rate was $72 \%$, with the 5 -year distant-metastasis-free survival rate approaching 90\%. "A European expert panel has recommended that patients achieving a complete clinical response should be given the option of "watch and wait' following CRT," Dr. Willett said.

Various ongoing watch-and-wait trials are currently underway. The randomized phase II OPRA trial is assessing 3-year DFS in patients with locally advanced disease treated with CRT + induction or consolidation chemotherapy and TME or nonoperative management. ${ }^{20}$ Another phase II study is underway at the Royal Marsden Hospital (NCT01047969).

\section{Omission of Radiation}

Ongoing studies are also evaluating the omission of radiation in select patients. A pilot study from Memorial Sloan Kettering Cancer Center enrolled 32 patients with stage II/III rectal cancers and nonthreatened circumferential margins whose treatment goal was sphincter preservation. ${ }^{21}$ After 6 cycles of FOLFOX + bevacizumab (only 2 patients had preoperative CRT), the R0 resection rate was $100 \%$, pathologic complete response rate was $25 \%$, 4-year local recurrence rate was $0 \%$, and 4 -year DFS rate was $84 \%$, and $91 \%$ of patients were alive at 4 years. "These are very interesting data showing quite a satisfactory outcome in selected patients, without CRT," Dr. Willett commented.

These data were the foundation for the phase III Alliance PROSPECT study (ClinicalTrials.gov identifier: NCT01515787), which is evaluating neoadjuvant FOLFOX with the selective use of combined modality CRT for patients with locally advanced disease undergoing low anterior resection with TME, where treatment intensity is chosen based on response to preoperative therapy. The study is accrued and the results "eagerly" awaited, Dr. Willett reported.

Disclosures: Dr. Venook has disclosed serving as a scientific advisor for Amgen Inc., Genentech, Inc., Merck \& Co., Inc., and Roche Laboratories, Inc. Dr. Willett has disclosed no relevant financial relationships.

Correspondence: Alan P. Venook, MD, UCSF Helen Diller Family Comprehensive Cancer Center, 1450 3rd Street, HD 376, Box 3111, San Francisco, CA 94143-3111. Email: Alan.Venook@ucsf.edu; and Christopher G. Willett, MD, Duke Cancer Institute, 30 Duke Medicine Circle, Room 05143 Morris, Box 3085, Durham, NC 27710.

Email: christopher.willett@duke.edu

\section{References}

1. Nojadeh JN, Behrouz Sharif S, Sakhinia E. Microsatellite instability in colorectal cancer. EXCLI J 2018;17:159-168.

2. Le DT, Kim TW, Van Cutsem E, et al. Phase II open-label study of pembrolizumab in treatment-refractory, microsatellite instability-high/ mismatch repair-deficient metastatic colorectal cancer: KEYNOTE-164 J Clin Oncol 2020;38:11-19.

3. Overman MJ, Lonardi S, Wong KYM, et al. Durable clinical benefit with nivolumab plus ipilimumab in DNA mismatch repair-deficient/microsatellite instability-high metastatic colorectal cancer. J Clin Oncol 2018;36:773-779.

4. Lenz HJ, Lonardi S, Zagonel V, et al. Subgroup analyses of patients with microsatellite instability-high/mismatch repair-deficient (MSI-H/dMMR) metastatic colorectal cancer treated with nivolumab plus low-dose ipilimumab as first-line therapy: two-year clinical update [abstract]. J Clin Oncol 2021;39(Suppl 6): Abstract 58

5. Andre T, Shiu KK, Kim TW, et al. Pembrolizumab versus chemotherapy for microsatellite instability-high/mismatch repair deficient metastatic colorectal cancer: the phase 3 KEYNOTE-177 study [abstract]. Presented at the 2020 ASCO Annual Meeting; May 29-31, 2020. Abstract LBA4.

6. Shiu KK, Andre T, Kim TW, et al. KEYNOTE-177: phase 2 randomized study of pembrolizumab versus chemotherapy for microsatellite instability-high advanced colorectal cancer [abstract]. J Clin Oncol 2021; 39(Suppl): Abstract 6.

7. Innocenti F, Ou FS, Qu X, et al. Mutational analysis of patients with colorectal cancer in CALGB/SWOG 80405 identifies new roles of microsatellite instability and tumor mutational burden for patient outcome. J Clin Oncol 2019;37:1217-1227.

8. Pfeiffer P, Yilmaz M, Moller S, et al. TAS-102 with or without bevacizumab in patients with chemorefractory metastatic colorectal cancer: an 
investigator-initiated, open-label randomized phase 2 trial. Lancet Oncol 2020;21:412-420.

9. Abraham JP, Magee D, Cremonlini C, et al. Clinical validation of a machine-learning-derived signature predictive of outcomes from first-line oxaliplatin-based chemotherapy in advanced colorectal cancer. Clin Cancer Res 2021:27:1174-1183.

10. Venook AP, Niedzwiecki D, Lenz HJ, et al. Effect of first-line chemotherapy combined with cetuximab or bevacizumab on overall survival in patients with KRAS wild-type advanced or metastatic colorectal cancer: a randomized clinical trial. JAMA 2017;317:2392-2401.

11. Marco MR, Zhou L, Patil S, et al. Consolidation mFOLFOX6 chemotherapy after chemoradiotherapy improves survival in patients with locally advanced rectal cancer: final results of a multicenter phase II trial. Dis Colon Rectum 2018;61:1146-1155.

12. Fokas $E$, Allgäuer M, Polat B, et al. Randomized phase II trial of chemoradiotherapy plus induction or consolidation chemotherapy as total neoadjuvant therapy for locally advanced rectal cancer: CAO/ARO/ AIO-12. J Clin Oncol 2019;37:3212-3222.

13. Conroy $\mathrm{T}$, Bosset JF, Etienne PL, et al. Neoadjuvant chemotherapy with FOLFIRINOX and preoperative chemoradiotherapy for patients with locally advanced rectal cancer (UNICANCER-PRODIGE 23): a multicentre, randomised, open-label, phase 3 trial [published online April 13, 2021] Lancet Oncol, doi: 10.1016/S1470-2045(21)00079-6

14. Bahadoer RR, Dijkstra EA, van Etten B, et al. Short-course radiotherapy followed by chemotherapy before total mesorectal excision (TME) versus preoperative chemoradiotherapy, TME, and optional adjuvant chemotherapy in locally advanced rectal cancer (RAPIDO): a randomised, open-label, phase 3 trial. Lancet Oncol 2021; 22:29-42.
15. Garcia-Aguilar J, Patil S, Kim JK, et al. Preliminary results of the organ preservation of rectal adenocarcinoma (OPRA) trial [abstract]. J Clin Oncol 2020;38(Suppl):Abstract 4008

16. Habr-Gama A, Gama-Rodrigues J, São Julião GP, et al. Local recurrence after complete clinical response and watch and wait in rectal cancer after neoadjuvant chemoradiation: impact of salvage therapy on local disease control. Int J Radiat Oncol Biol Phys 2014;88:822-828.

17. Martens $\mathrm{MH}$, Maas M, Heijnen LA, et al. Long-term outcome of an organ preservation program after neoadjuvant treatment for rectal cancer. J Natl Cancer Inst 2016;108:djw171.

18. Appelt AL, Pløen J, Harling $\mathrm{H}$, et al. High-dose chemoradiotherapy and watchful waiting for distal rectal cancer: a prospective observational study. Lancet Oncol 2015;87:919-927.

19. Fernandez LM, Sao Juliao GP, Figueiredo NL, et al. Conditional recurrence-free survival of clinical complete responders managed by watch and watch after neoadjuvant chemotherapy for rectal cancer in the International Watch \& Wait Database: a retrospective, international, multicentre registry study. Lancet Oncol 2020;22:43-50.

20. Smith JJ, Chow OS, Gollub MJ, et al. Organ preservation in rectal adenocarcinoma: a phase II randomized controlled trial evaluating 3-yea disease-free survival in patients with locally advanced rectal cancer treated with chemoradiation plus induction or consolidation chemotherapy, and total mesorectal excision or nonoperative management. BMC Cancer 2015;15:767.

21. Schrag D, Weiser MR, Goodman KA, et al. Neoadjuvant chemotherapy without routine use of radiation therapy for patients with locally advanced rectal cancer: a pilot trial. J Clin Oncol 2014;32:513-518. 